\title{
Function of small-fiber assessed with contact heat evoked potentials in patients with diabetic neuropathy
}

\author{
Qian $\mathrm{Li}^{1-4}$, Miao Yü ${ }^{5}$ Zhecheng Zhang ${ }^{1-4}$ and Jing Zhang ${ }^{1-4 *}$ \\ ${ }^{1}$ Department of Neurology, The Third Central Hospital of Tianjin, 83 Jintang Road, Hedong District, Tianjin, China \\ ${ }^{2}$ Tianjin Key Laboratory of Extracorporeal Life Support for Critical Diseases, Tianjin, China \\ ${ }^{3}$ Artificial Cell Engineering Technology Research Center of Public Health Ministry, Tianjin, China \\ ${ }^{4}$ Tianjin Institute of Hepatobiliary Disease,Tianjin, China \\ ${ }^{5}$ Department of Neurology,Tianjin Huanhu Hospital, Jizhao Road, Jinnan District, Tianjin, China
}

\begin{abstract}
Background: We evaluated sensitivity of contact heat evoked potentials (CHEPs) compared with sympathetic skin response (SSR) and Toronto Clinical Scoring System (TCSS) in diabetic neuropathy (DN) at early stage and analyze the characteristics of small fibers injury.

Methods: A case-control study was conducted in $100 \mathrm{DN}$ patients and 50 controls. A peak stimulating temperature of $51^{\circ} \mathrm{C}$ was applied to the mandibular region, region above the eyebrow, lower limb an forearm. CHEPs parameters were recorded by the $\mathrm{C}_{\mathrm{z}}$ point. The SSR examination and TCSS were also performed.

Results: There were significant differences in the median of the ratio of $\mathrm{N}$-wave latency in mandibular region to that in region above the eyebrow between the DM group and controls $(Z=-3.441, \mathrm{P}=0.001)$. There were also significant differences in the median of the ratio of $\mathrm{N}$ wave amplitude in forearm to that in the lower limb between the DM group and controls $(Z=-3.441, \mathrm{P}<0.01)$. The latency of the DMN group was longer than that of the controls after stimulation of the mandibular region. When we stimulated the region above the eyebrow, lower limb and forearm at $51^{\circ} \mathrm{C}$, the DMN group exhibited lower $\mathrm{N}-\mathrm{P}$ amplitude and more prolonged $\mathrm{N}$ wave latency compared to those in controls. On the other side, when the region above eyebrow and lower limb was stimulated, the SSRN group had lower N-P amplitude and more prolonged $\mathrm{N}$ wave latency compared to controls.
\end{abstract}

Conclusion: Compared with TCSS and SSR, CHEPs had higher sensitivity in the detection of DN at early stage.

Abbreviations: CHEPs: Contact heat evoked potentials; SSR: Sympathetic skin response; TCSS: Clinical scoring system; DN: Diabetic neuropathy; DM: Diabetes mellitus; IGT: Impaired glucose tolerance; SSRA: SSR abnormal group; SSRN: SSR normal group; EEG: Electroencephalogram.

\section{Introduction}

Diabetic neuropathy (DN) can affect the nervous system and is one of the most common complications of diabetes mellitus (DM) [1]. Neurofibrillary damage can be the initial symptom of DM [2], which can be seen at the stage of impaired glucose tolerance (IGT). Thus, developing new approaches for detecting DN at early stage is of great significance. At early stage, the pathological changes occur in the myelinated $\mathrm{A} \delta$ fibers and unmyelinated $\mathrm{C}$ fibers, which is a pathological processes called diabetic small-fiber neuropathy (DN) [3].

Although DN is hard to be diagnosed due to the lack of corresponding clinical symptoms and signs, electrophysiological evaluation can provide objective standard for its diagnosis and treatment [4]. The Toronto Scoring System (TCSS), a standard method for detecting and monitoring DN [5], mainly evaluates the neurological damage of patients according to clinical manifestations and physical examination. The validity of the TCSS score can be verified by comparing with the results of electrophysiological examination and sural nerve morphological examination [6]. In addition, the sympathetic skin response (SSR) can reflect the function of unmyelinated C fibers after sympathetic joints and detect autonomic neuropathy in patients with DM at early stages [7]. Recent research have evaluated the A $\delta$ fibers function of the spinal nerve using the contact heat evoked potentials (CHEPs) $[1,8,9]$. But the involvement of cranial small-fiber still need to be further explored. Our aim was to assess the feasibility of CHEP for detection DN at early stage and analyze the characteristics of smallfiber injury in both of the cranial nerves and spinal nerves.

\section{Methods}

\section{Study design and study participants}

We conducted a case-control study in the Third Central Hospital of Tianjin.100 DM patients and 50 healthy controls were recruited by Department of Metabolism, Third Central Hospital of Tianjin between June 2015 and January 2017. According to the 1999 WHO Diabetes Diagnostic Criteria, 60 patients with confirmed diagnosis included 47 males and 53 females, age 43-77 years with DM course from 4 months

${ }^{\star}$ Correspondence to: Jing Zhang, Department of Neurology, The Third Central Hospital of Tianjin, 83 Jintang Road, Hedong District, Tianjin, China, Tel: +8622-84112177; E-mail: yadibaby1019@163.com

Key words: diabetic neuropathy Contact heat evoked potentials Skin sympathetic response, Toronto clinical scoring system small-fiber function

Received: April 09, 2020; Accepted: April 19, 2020; Published: April 25, 2020 
to 21 years. The exclusion criteria were: 1 . History of ischemic or hemorrhagic cerebrovascular disease; 2 . Neck and lumbar vertebrae lesions (neural root compression, spinal stenosis); 3. Toxic, infectious, nutritional, immune peripheral neuropathies such as alcoholic peripheral neuropathy, Guillain-Barre syndrome, toxic peripheral neuropathy etc; 4 . Severe arteriovenous vascular disease, such as venous thrombosis, lymphangitis, etc; 5 . Neuropathy caused by metabolic toxicities from renal insufficiency; 6 . Foot ulcers, infections and edema; 7. Single neuropathy, such as carpal tunnel syndrome, elbow syndrome, etc; 8 . Peripheral neuropathy caused by others than diabetes. The sex $(\chi 2=0.143)$ and age $(t=1.281)(p>0.05)$ were no significant difference between the DM group and the controls. The skin of the tested site was intact. All of subjects signed informed consent forms before the procedure.

According to the TCCS score, the DM group were divided into two subgroups: DM patients with TCCS $>5$ as DMA and DM patients with TCCS $\leq 5$ as DMN. According to the SSR results, the DM patients were divided into the SSR abnormal group (SSRA) and the SSR normal group (SSRN). SSR abnormality refers to the prolongation of the initiation latency or reduction of amplitude during SSR examination from any limb of DM patients.

\section{TCSS examination}

Subjects were graded according to neuropathy severity using 6 symptom scores (lower extremity pain, numbness, acupuncture, fatigue, walking imbalance and upper limb symptoms), 8 reflex scores (the presence or absence pf bilateral knee and ankle reflexes, each graded as absent, reduced, or normal), and 5 physical examination scores (the presence or absence of pinprick, temperature, light touch, vibration, and position sense) for a total of 19 possible points. Grading was stratified. such that 5 indicated no neuropathy,6-8 indicated mild neuropathy, 9-11 indicated moderate neuropathy, and 12 indicated severe neuropathy [10] TCSS is reliable in diagnosing DN with advantages of simple and comprehensive, which can be used for evaluating both large and small nerve fiber functions [11].

\section{SSR examination}

Using saddle electrode, the stimulation was placed on wrist and the medial malleolus. Single electrical stimuli was performed suddenly. Stimulation was applied at $50 \mathrm{~mA}$. The stimuli were repeated 2 times with a longer than $60 \mathrm{~s}$ for each stimulation to avoid habituation of response. The recording electrodes were attached to the palm of the hands as well as to the sole of the feet. The reference electrodes were attached to the centre of the dorsum of the hands and the dorsum of the feet. The potentials associated with sweat glands activity can be elicited and the skin sympathetic response of the extremities can be recorded. The normal standard value refers to the reference [12].

\section{CHEP examination}

We performed CHEP examination instrument setting and procedure according to previous descriptions by Zhang et al. [13]. The thermode (27 $\mathrm{mm}$ in diameter) consists of an external fast-heating foil with a heating rate of $70^{\circ} \mathrm{C} / \mathrm{s}$. During the examination, subjects lay with their eyes closed in a quiet room with temperature around $22 \sim 25^{\circ} \mathrm{C}$. Repeated stimulation applied on the right side of mandibular region, the right side of supraorbital region, right lower leg and distal side of the right forearm with a stimulator for 5 to 8 times with an interval from 15 to $25 \mathrm{~s}$. At the peak temperature $\left(51^{\circ} \mathrm{C}\right)$ [14], according to the international electroencephalogram (EEG) 10-20 system, the recording electrode was placed at the $\mathrm{Cz}$ point and the reference electrode was placed at Fpz. We rated the intensity of the stimulation after each trial of the stimulation according to visual analog scale (VAS) [15].

\section{Statistical analysis}

Statistical analysis was performed using the SPSS 16.0 statistical software. CHEP latency and amplitude were in accordance with the normal distribution, expressed as mean \pm standard deviation $( \pm s)$. Other data were skewed distribution, presenting with median (quartile spacing) $[\mathrm{M}(\mathrm{Q})]$. Student $\mathrm{t}$ test was applied for comparison of independent samples between two groups. We applied the MannWhitney $\mathrm{U}$ test for comparison between the two groups. The CHEP amplitude and latency between three groups were compared with one-way analysis of variance, and the comparison between groups was analysed by LSD method. $\mathrm{P}<0.05$ was considered statistically significant.

\section{Results}

\section{Comparing the results of TCSS scoring}

The median TCSS score (interquartile range) in the DM group was 5 (4 to 10), the median TCSS score (interquartile range) in the DM group is significantly higher than that in controls. In addition, the abnormal rate of TCSS score (TCSS $\geq 6$ points) in DM group was $41.0 \%$, suggesting that TCSS is a validated tool to detect DN.

\section{Comparing the results of SSR examination}

In the SSR examination, it was abnormal in $74 \%$ diabetic patients. Diabetic patients had significantly prolonged latency in lower limbs and lower amplitude in both the upper and lower limbs compared with the controls (Table 1).

\section{Comparing the results of CHEP cranial nerve test}

\section{Comparison of CHEP and VAS scores in the mandibular and periorbital region between DM and controls}

Patients with DM had lower VAS than controls $(\mathrm{Z}=6.189, \mathrm{p}<0.01$; $\mathrm{Z}=6.411, \mathrm{p}<0.01)$. Evoked potentials could be recorded by stimuli applied to the mandibular and region above the eyebrow at $51^{\circ} \mathrm{C}$. There were significant differences in the $\mathrm{N}$-wave latency and the $\mathrm{N}$-P amplitude between $\mathrm{DM}$ group and controls. ( $\mathrm{N}$-wave latency: $378.77 \pm 36.82$ vs $354.42 \pm 23.36 \mathrm{~ms}, \mathrm{p}<0.01 ; 383.26 \pm 36.46$ vs $342.66 \pm 17.30 \mathrm{~ms}, \mathrm{p}<0.01$; $\mathrm{N}-\mathrm{P}$ wave amplitudes: $48.62 \pm 18.80$ vs $60.37 \pm 10.84$, $\mathrm{p}<0.01 ; 47.38 \pm 16.20$ vs62.32 $\pm 17.75 \mu v, \mathrm{p}<0.01$ ) (Figure 1 ).

Table 1. SSR in controls and diabetics $[\mathrm{M}(\mathrm{Q}) /]$

\begin{tabular}{|c|c|c|c|c|c|}
\hline \multirow[b]{2}{*}{-} & \multirow{2}{*}{$\mathbf{N}$} & \multicolumn{2}{|c|}{ Hand } & \multicolumn{2}{|c|}{ Foot } \\
\hline & & Latency(ms) & Amplitude $(\mu V)$ & Latency(ms) & $\operatorname{Amplitude}(\mu V)$ \\
\hline $\mathrm{DM}$ & 100 & $1401(1321,1470)$ & $500(373,558)$ & $2012(1922,2188)$ & $193(82,379)$ \\
\hline Control & 50 & $1390(1288,1451)$ & $560(513,654)$ & $1900(1784,2003)$ & $532(504,614)$ \\
\hline$Z$ & & -0.865 & 4.768 & -3.986 & 7.514 \\
\hline$P$ & & 0.387 & $<0.01$ & $<0.01$ & $<0.01$ \\
\hline SSR absent rate & & $10 \%$ & $33 \%$ & $20 \%$ & $72 \%$ \\
\hline
\end{tabular}



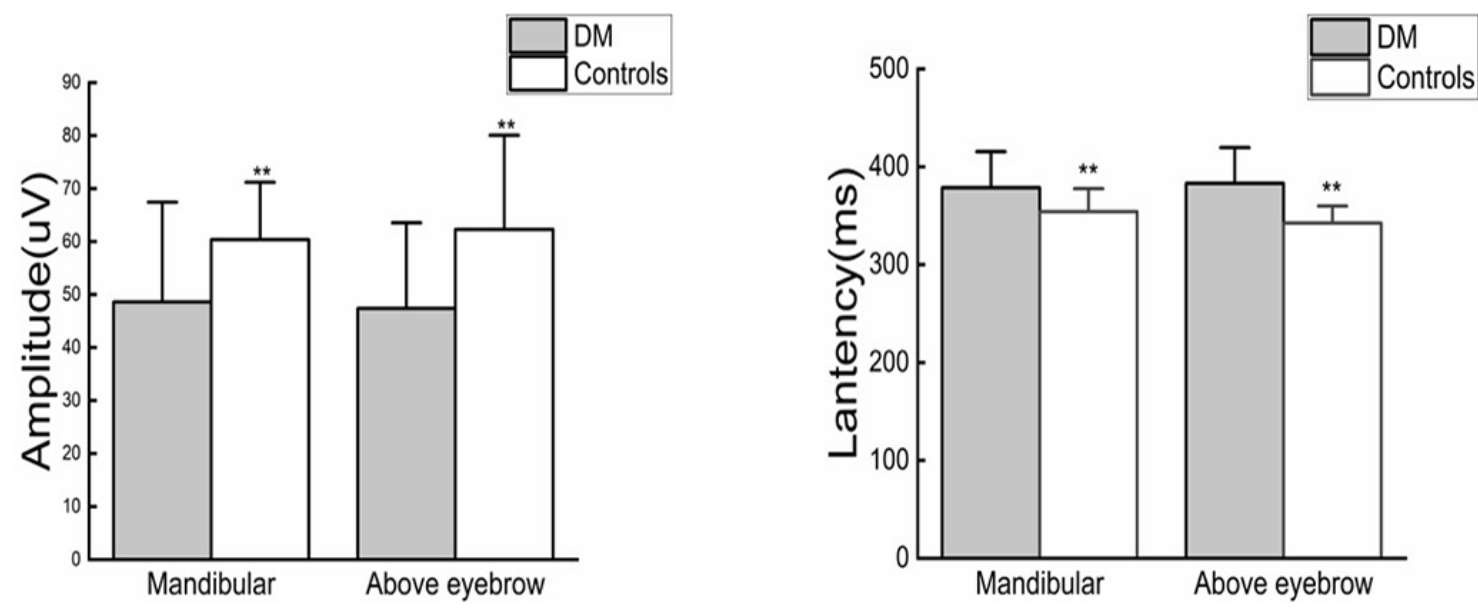

Figure 1. Comparison of N-wave latency ratio and N-P wave amplitude ratio in mandibular region and region above eyebrow between DM and controls

Comparison of $\mathrm{N}$ wave latency ratio and $\mathrm{N}-\mathrm{P}$ wave amplitude ratio in mandibular region and region above eyebrow between $\mathrm{DM}$ and controls

There were significant differences in the median of the ratio of N-P wave latency in mandibular region to that in the region above eyebrow between the DM group and controls when thermal stimulation was applied $(\mathrm{Z}=-3.441, \mathrm{p}=0.001)$ (Figure $2 \mathrm{~A})$. However, there was no significant difference in the median of the ratio of $\mathrm{N}$ wave amplitude in mandibular region to that in region above the eyebrow between the DM group and controls $(\mathrm{Z}=0.371, \mathrm{p}=0.711)$ (Figure $2 \mathrm{~B})$.

\section{CHEP spinal nerve examination}

Comparison of CHEP and VAS scores in lower limb and forearm stimulation between DM and control group

Patients with DM had significantly lower VAS than controls $(\mathrm{Z}=3.695, \mathrm{P}<0.01 ; \mathrm{Z}=2.887, \mathrm{p}<0.01)$. At temperature of $51^{\circ} \mathrm{C}$, the forearm and lower limb from all patients were stimulated, and CHEPs were recorded at the $\mathrm{CZ}$ positions of the standard 10-20 EEG montage. Thermal simulation of the forearm and lower limb resulted in significant differences in CHEP $\mathrm{N}$ wave latency and $\mathrm{N}-\mathrm{P}$ wave amplitude between the DM group and controls $(\mathrm{P}<0.01)$ (Figure $2 \mathrm{C})$.

Comparison of CHEP N-wave latency ratio and N-P wave amplitude ratio in lower limb and forearm stimulation between DM and control group

There were significant differences in the median of the ratio of N-P wave amplitude in forearm to that in the lower limb between the DM group and controls $(\mathrm{Z}=-3.441, \mathrm{p}<0.01)$ (Figure 2D). However, there was no significant difference in the median of the ratio of $\mathrm{N}$ wave latency in forearm to that in the lower limb between the DM group and controls $(\mathrm{Z}=0.371, \mathrm{p}>0.05)$ (Figure 3).

\section{Comparison of CHEP results according to TCSS grouping}

Comparison of CHEP results of cranial nerves according to TCSS grouping

Thermal stimulation of the skin of the mandibular region at the temperature of $51^{\circ} \mathrm{C}$ resulted in prolonged $\mathrm{N}$-wave latency in the DMN group than controls, but there was no significant difference in $\mathrm{N}-\mathrm{P}$ amplitude between them. When thermal stimulation applied to the region above eyebrow at $51^{\circ} \mathrm{C}, \mathrm{DMN}$ group had more prolonged $\mathrm{N}$ wave latency and lower amplitude of N-P wave compared with the controls. Besides, DMA group had prolonged $\mathrm{N}$-wave latency and lower amplitude of N-P wave than the DMN group (Table 2).

Comparison of CHEP results of spinal nerve according to TCSS grouping

When the forearm and the lower limb was stimulated at $51^{\circ} \mathrm{C}$, the DMN group had lower N-P wave amplitude and prolonged latency of $\mathrm{N}$-wave compared with controls. In addition, the amplitude of N-P wave in DMA group was significantly lower than that in the DMN group (Table 3 ).

\section{Comparison between results from CHEP groups according to SSR grouping}

Comparison of cranial nerve CHEP results according to SSR grouping

When thermal stimulation was applied to the mandibular region, there were no significant differences in N-wave latency and N-P amplitude between the SSRN group and controls. However, the thermal simulation of region above eyebrow resulted in significant differences in N-wave latency and N-P amplitude between the SSRN group and controls. Besides, the SSRA group had more prolonged $\mathrm{N}$-wave latency compared with the SSRN group, while there was no significant difference in the N-P wave amplitude between the two groups (Table 4).

\section{Comparison of spinal nerve results according to SSR grouping}

When thermal simulation was applied to the forearm, there were no significant differences in N-wave latency and N-P amplitude between the SSRN group and controls. However, when the lower limb was stimulated, the SSRN group had longer $\mathrm{N}$-wave latency and lower $\mathrm{N}-\mathrm{P}$ wave amplitude than that in controls. Furthermore, the amplitude of N-P wave in SSRA group was lower than that of SSRN group. The difference was statistically significant (Table 5).

\section{Discussion}

In this study we found that CHEPs had highly sensitivity in the early detection of small-fiber damage in DN. The results suggest that the first branch of the trigeminal nerve injury may be more severe than the third one. Moreover, the spinal nerve damage is length dependent which are axonal injury predominantly.

DN normally involves the small-fiber function at early stage [16], which manifests as pain, burning paresthesia, at the distal of 
A

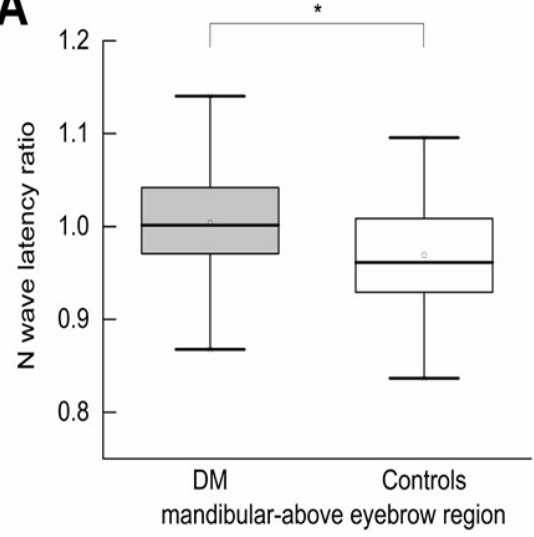

C

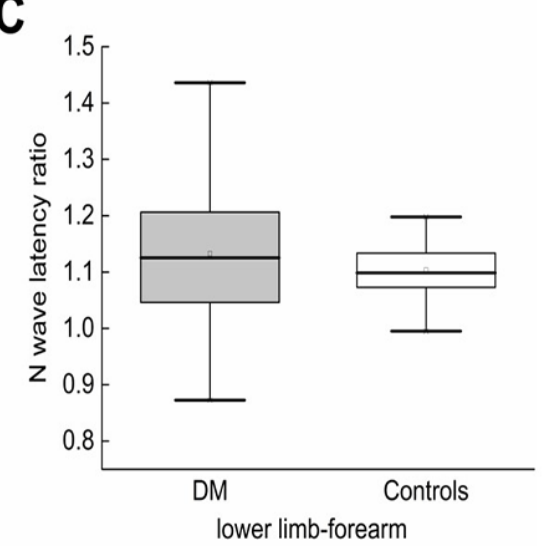

B

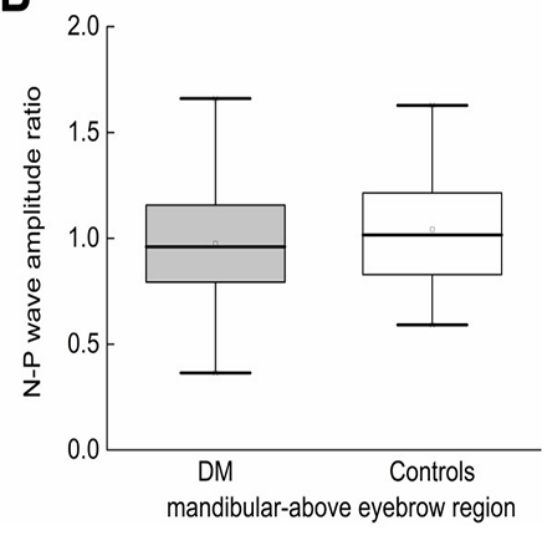

D

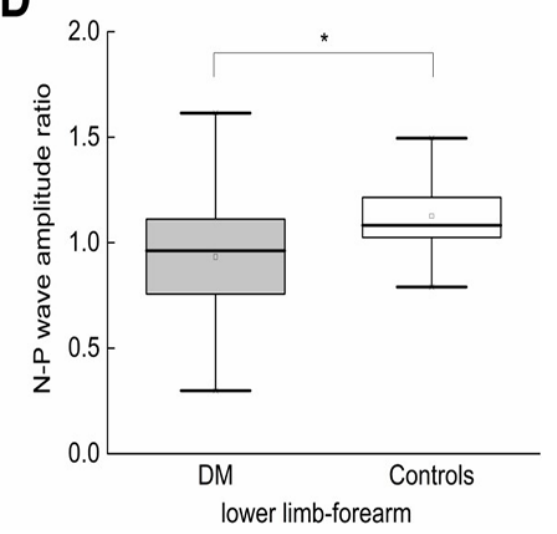

Figure 2. (A) N wave latency ratio in mandibular region to region above eyebrow between DM and controls. (B) N-P wave amplitude ratio in mandibular region to region above eyebrow between DM and controls. (C) N wave latency ratio in lower limbs to forearm stimulation between DM and controls. (D) N-P wave amplitude ratio in lower limbs to forearm stimulation between DM and controls

A

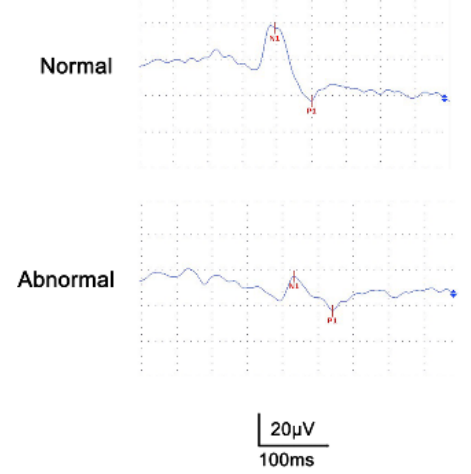

B

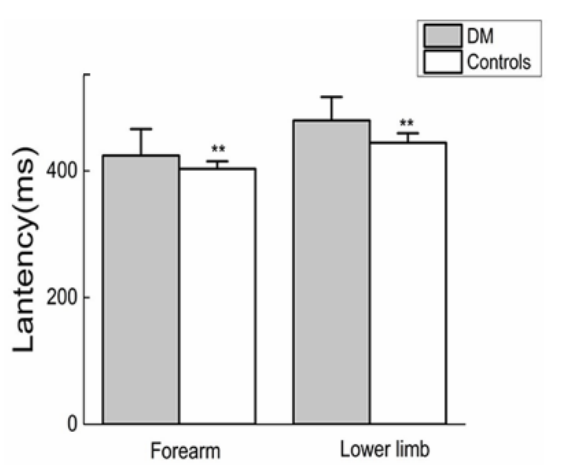

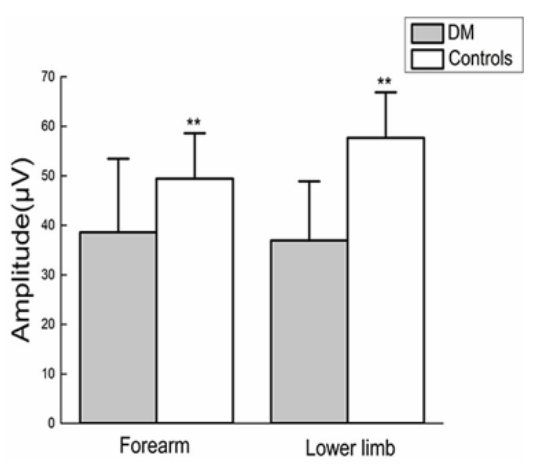

Figure 3. (A)When we applied stimuli in upper limb, DM patients had more prolonged N-wave latency and lower N-P amplitude than controls.(B) Comparison of N-wave latency ratio and $\mathrm{N}-\mathrm{P}$ wave amplitude ratio in right forearm and right lower limb between DM and controls 
Table 2. CHEP results of cranial nerves according to TCSS

\begin{tabular}{|c|c|c|c|c|c|c|c|c|c|}
\hline & \multirow{2}{*}{$\mathbf{N}$} & \multicolumn{4}{|c|}{ Mandibular } & \multicolumn{4}{|c|}{ Above eyebrow } \\
\hline & & Latency(ms) & t & Amplitude $(\mu \mathrm{V})$ & t & Latency(ms) & t & Amplitude $(\mu \mathrm{V})$ & t \\
\hline DMA & 41 & $389.10 \pm 33.71$ & $2.664 *$ & $40.01 \pm 12.90$ & -4.611 & $394.49 \pm 40.05$ & $3.060^{*}$ & $43.15 \pm 15.90$ & - \\
\hline DMN & 59 & $371.59 \pm 37.45$ & 5.093 & $54.60 \pm 19.99$ & -5.504 & $375.46 \pm 31.81$ & 8.043 & $50.32 \pm 15.89$ & $2.133^{*}$ \\
\hline Controls & 50 & $354.42 \pm 23.36$ & 2.764 & $60.37 \pm 10.84$ & -1.931 & $342.66 \pm 17.30$ & 5.579 & $62.32 \pm 17.75$ & -5.504 \\
\hline F & & 13.011 & & 20.142 & & $34.04 *$ & & 15.889 & -3.777 \\
\hline
\end{tabular}

Table 3. CHEP results of spinal nerves according to TCSS

\begin{tabular}{|c|c|c|c|c|c|c|c|c|c|}
\hline & \multirow{2}{*}{$\mathbf{N}$} & \multicolumn{4}{|c|}{ Forearm } & \multicolumn{4}{|c|}{ Lower limb } \\
\hline & & Latency(ms) & $\mathbf{t}$ & Amplitude $(\mu V)$ & $\mathbf{t}$ & Latency(ms) & $\mathbf{t}$ & Amplitude $(\mu V)$ & $\mathbf{t}$ \\
\hline DMA & 41 & $427.02 \pm 39.40$ & $0.750 \mathrm{a}$ & $34.17 \pm 14.94$ & -2.86 & $479.80 \pm 33.29$ & 0.154 & $33.56 \pm 10.35$ & -2.576 \\
\hline $\mathrm{DMN}$ & 59 & $421.71 \pm 43.50$ & $3.285 \mathrm{~b}$ & $41.69 \pm 14.11$ & -5.599 & $478.81 \pm 39.74$ & 5.363 & $39.28 \pm 12.53$ & -10.461 \\
\hline Controls & 50 & $402.90 \pm 11.77$ & $2.808 \mathrm{c}$ & $49.43 \pm 9.18$ & -3.112 & $444.08 \pm 15.02$ & 5.715 & $57.64 \pm 9.22$ & -8.742 \\
\hline $\mathrm{F}$ & & 6.324 & & 15.757 & & 20.593 & & 63.171 & \\
\hline
\end{tabular}

Table 4. CHEP results of carnial nerves according to SSR

\begin{tabular}{|c|c|c|c|c|c|c|c|c|c|}
\hline & \multirow{2}{*}{$\mathbf{N}$} & \multicolumn{4}{|c|}{ Mandibular } & \multicolumn{4}{|c|}{ Above eyebrow } \\
\hline & & Latency(ms) & $\mathbf{t}$ & Amplitude $(\mu \mathrm{V})$ & $\mathbf{t}$ & Latency(ms) & $\mathbf{t}$ & Amplitude $(\mu \mathrm{V})$ & $\mathbf{t}$ \\
\hline SSRA & 74 & $384.49 \pm 38.77$ & $3.003 *$ & $45.16 \pm 16.01$ & -3.653 & $388.72 \pm 38.02$ & $3.006^{*}$ & $46.40 \pm 16.35$ & -0.989 \\
\hline SSRN & 26 & $362.50 \pm 24.61$ & 5.113 & $58.44 \pm 22.7 \mathrm{t}$ & -5.211 & $367.73 \pm 26.56$ & 8.217 & $50.17 \pm 15.72$ & -5.199 \\
\hline Controls & 50 & $354.42 \pm 23.36$ & 1.04 & $60.37 \pm 10.84$ & -0.502 & $342.66 \pm 17.30$ & 3.387 & $62.32 \pm 17.75$ & -3.004 \\
\hline $\mathrm{F}$ & & 14.089 & & 15.736 & & 33.824 & & 13.782 & \\
\hline
\end{tabular}

Table 5. CHEP results of spinal nerves according to SSR

\begin{tabular}{|c|c|c|c|c|c|c|c|c|c|}
\hline & \multirow{2}{*}{$\mathbf{N}$} & \multicolumn{4}{|c|}{ Forearm } & \multicolumn{4}{|c|}{ Lower limb } \\
\hline & & Latency(ms) & t & Amplitude $(\mu \mathrm{V})$ & t & Latency(ms) & t & Amplitude $(\mu \mathrm{V})$ & t \\
\hline SSRA & 74 & $427.49 \pm 40.63$ & $1.755^{*}$ & $35.90 \pm 13.30$ & -3.591 & $484.23 \pm 36.95$ & $2.740^{*}$ & $35.44 \pm 11.91$ & -2.289 \\
\hline SSRN & 26 & $413.65 \pm 43.96$ & 3.886 & $46.33 \pm 16.53$ & -5.802 & $464.96 \pm 34.15$ & 7.11 & $41.17 \pm 11.30$ & -11.046 \\
\hline Controls & 50 & $402.90 \pm 11.77$ & 1.287 & $49.43 \pm 9.18$ & -1.007 & $444.08 \pm 15.02$ & 2.8 & $57.64 \pm 9.22$ & -6.205 \\
\hline $\mathrm{F}$ & & 7.687 & & 18.469 & & 25.382 & & 61.929 & \\
\hline
\end{tabular}

the extremities and insomnia. DN seriously affect the quality of life [17]. Moreover, DN patients usually have no obvious clinical symptoms and signs, that is why diagnosis of DN is difficult by routine electrophysiological examination. Due to its high rates of delayed and wrong diagnosis, most of patients had missed their opportunities of treatment. Therefore, developing new approaches for detecting DN at early stage is of great significance.

The incidence of cranial nerve injury in patients with DM is only $0.32 \%$ to $1.0 \%$ due to atypical symptoms and lack of specific detection methods $[18,19]$. This study is the first to demonstrate the feasibility of CHEP as a clinical examination for analysing the characteristics of cranial nerves injury. When we stimulated mandibular region and region above the eyebrow, patients with DM had lower VAS than controls $(\mathrm{Z}=6.189, \mathrm{p}<0.01 ; \mathrm{Z}=6.411, \mathrm{p}<0.01)$. There were significant differences in the N-wave latency and the N-P amplitude between DM group and controls. These findings demonstrate that DM patients have thermal hypoalgesia of the face and damage to both of the axon and myelin sheath in trigeminal myelinated A $\delta$ fibers,. The results are consistent with previous studies by Agostino [20], which means application of CHEP may increase the detection rate of cranial nerve injury.

There were significant differences in the median of the ratio of $\mathrm{N}$ wave latency in mandibular region to that in the region above eyebrow between the DM group and controls. The latency was more prolonged when the region above eyebrow was stimulated compared with that when mandibular region was stimulated, indicating the damage of the first is more severe than the third branch of trigeminal nerve in DN patients. In addition, the first branch of trigeminal nerve demyelinating damage is severe than the third one, we inferred that it may result from their different anatomy, nerve route and blood supply. This is consistent with previous case reports in which manifested as the periocular hypoalgesia or periocular pain [21].

Some studies regard that the prolonged SSR latency as a sign of conduction delays of sympathetic nerve, and the reduced amplitude suggest impaired function of unmyelinated fibers that innervate sweat glands [7]. In the SSR examination, DM patients mainly showed a decrease in SSR amplitude and the abnormal rate of SSR in the lower limbs was higher than that in the upper limbs, on the other hand, there were significant differences in the median of the ratio of N-P wave amplitude in the forearm to the N-P wave amplitude in the lower limbs between the DM group and controls. The results suggested that sympathetic post-ganglionic C-fibers damage in lower limbs is severe than upper limbs in DN patients with diabetes which are affected in a length-dependent pattern [22].

Comparison CHEP results according to TCSS grouping revealed that small fibers damage of cranial sensory nerve (trigeminal nerve) in DN patients. Furthermore, CHEP can detect cranial nerve and spinal nerve fibers damage at early stage when the TCSS score was normal. Tu MC [23] and colleagues reported that a patient with multiple cranial neuropathy suspected the presence of periocular acupuncture hyposensitivity, but the BR results were normal. We inferred that cranial sensory nerve fibers damage may already exist when the electrophysiological examination of large myelinated fibers was normal. CHEP could detect DN cranial sensory nerve fibers injury at earlier stage. 
Comparison CHEP results of cranial nerves according to SSR revealed that the thermal simulation of region above eyebrow resulted in significant differences in N-wave latency and $\mathrm{N}-\mathrm{P}$ amplitude between the SSRN group and controls, suggesting that there was small-fiber damage in both of the cranial and autonomic nerves in DN and CHEP may reflect the damage to the first branch of trigeminal nerve when SSR was normal. A prolongation in CHEP latency was common in DN patients, a factor contributing to this could be cranial A $\delta$-fiber damage is prior to the postganglionic autonomic C-fiber damage or both of them are involved.

Comparison CHEP results of spinal nerves according to SSR revealed that spinal small-fiber damage in the lower limbs is more severe than that in the upper limbs. It is length dependent with axonal injury. Al Moallem et al. [24] demonstrated that the measurement of SSR latency is an objective method to determine the conduction in many nerve pathways, which can be used to detect the subclinical lesions in sympathetic nervous system among DN patients. Our data confirmed that CHEP can detect small-fiber damage earlier than SSR [13],which is consistent with previous reports [7].

\section{Conclusions}

In conclusion, DN can affect the cranial nerve, spinal nerve and autonomic nerve. CHEPs can effectively detect DN at early stage even when TCSS and SSR are normal. Moreover, we found the trigeminal nerve injury may be non-length dependent. The first branch is severe than the third one and is primarily demyelinating at early stage. Spinal small-fiber damage in the lower limbs is more severe than that in the upper limbs. It is length dependent with axonal injury. The results of this study demonstrate the potential clinical application of CHEP.

\section{Declaration}

\section{Authors' contributions}

Qian $\mathrm{Li}$ and Miao Yu are responsible for the study design, data collection. Qian Li drafted the initial manuscript, reviewed and revised the manuscript, and approved the final manuscript as submitted. Miao Yu performed nerve conduction studies in patients, Zhecheng Zhang participated in the conception of the study and helped to interpret the data. Jing Zhang conceived the study and participated in its design and co-ordination, interpretation, and completion of the manuscript. All authors read and approved the manuscript.

\section{Acknowledgements}

Not applicable

\section{Funding}

This work was supported by the Major science and technology projects for the prevention and treatment of major diseases from Tianjin science and technology commission (19ZXDBSY00100).

\section{Conflicts of interest}

The authors declare that they have no competing interests in the study.

\section{Ethics approval and consent to participate}

Written informed consents were obtained from the participants for publication of this study. All studies have been approved by the appropriate ethics committee and have, therefore, been performed in accordance with the ethical standards laid down in the 1964 Declaration of Helsinki and its later amendments.

\section{Consent for publication}

Written informed consents were obtained from the participants for publication of this study and any accompanying images. The participants agreed to publish this study via written consents.

\section{References}

1. Cheng Y, Liu J, Luan Y, Liu Z, Zhai Q (2019) Sarm1 gene deficiency attenuates diabetic peripheral neuropathy in mice. Diabetes 68: db181233. [Crossref]

2. Peterson M, Pingel R, Lagali N, Dahlin LB, Rolandsson O (2017) Association between $\mathrm{HbA}(1 \mathrm{c})$ and peripheral neuropathy in a 10-year follow-up study of people with normal glucose tolerance, impaired glucose tolerance and Type 2 diabetes. Diabetic Medicine 34: 1756-64. [Crossref]

3. Di Stefano G, La Cesa S, Leone C, Pepe A, Galosi E, et al. (2017) Diagnostic accuracy of laser-evoked potentials in diabetic neuropathy. Pain 158: 1100-1107.

4. Kamel JT, Vogrin SJ, Knight-Sadler RJ, Willems NK, Seiderer L, et al. (2015) Combining cutaneous silent periods with quantitative sudomotor axon reflex testing in the assessment of diabetic small fiber neuropathy. Clinical Neurophysiology 126: $1047-1053$

5. Hu H, Li H, Zheng FP, Cheng Y, Miao (2012) A comparison of clinical effectiveness of different neuropathy scoring systems in screening asymptomatic diabetic peripheral neuropathy. Zhonghua Nei Ke Za Zhi 51: 13-17. [Crossref]

6. Abraham A, Barnett C, Katzberg HD, Lovblom LE, Perkins BA (2018) Toronto clinical neuropathy score is valid for a wide spectrum of polyneuropathies. European Journal of Neurology 25: 484-490.

7. Tiftikcioglu BI, Bilgin S, Duksal T, Kose S, Zorlu Y (2016) Autonomic neuropathy and endothelial dysfunction in patients with impaired glucose tolerance or type 2 diabetes mellitus. Medicine 95: e3340.

8. Wong MC, Chung JW (2011) Feasibility of contact heat evoked potentials for detection of diabetic neuropathy. Muscle \& Nerve 44: 902-906.

9. Parson HK, Nguyen VT, Orciga MA, Boyd AL, Casellini CM (2013) Contact heatevoked potential stimulation for the evaluation of small nerve fiber function. Diabetes Technology \& Therapeutics 15: 150-157.

10. Lou DJ, Zhu QQ, Xu-Wei SI (2013) Application of toronto clinical scoring system in screening diabetic peripheral neuropathy in T2DM patients. Chinese Journal of Diabetes 5: 56-61

11. Liu F, Mao JP, Yan X (2008) Toronto clinical scoring system in diabetic peripheral neuropathy. Journal of Central South University Medical sciences 33: 1137-1141.

12. Nazhel B, Yetkin I, Irkeç C, Koçer B (2002) Sympathetic skin response in diabetic neuropathy. Electromyography and Clinical Neurophysiology 42: 181-185.

13. Liu N, Zhang J (2018) Impact of impaired glucose regulation on the functions of large and small fibers of peripheral nerves. Turk J Med Sci 48: 1207-1213. [Crossref]

14. Warbrick T, Derbyshire SW, Bagshaw AP (2009) Optimizing the measurement of contact heat evoked potentials. Journal of Clinical Neurophysiology 26: 117-1122.

15. Sung YT, Wu JS (2018) The Visual analogue scale for rating, ranking and pairedcomparison (VAS-RRP): A new technique for psychological measurement. Behavior Research Methods 50: 1694-715.

16. Obayashi K, Ando Y (2014) Diagnosis of small-fiber neuropathy using various autonomic function tests. Rinsho Shinkeigaku 54: 1044-1046. [Crossref]

17. Gerawarapong C (2015) Association of peripheral autonomic neuropathy and sympathetic skin response in the patients with diabetic polyneuropathy: A pilot study in Thailand. Journal of the Medical Association of Thailand 98: 1222-1230.

18. Al Kahtani ES, Khandekar R, Al-Rubeaan K, Youssef AM, Ibrahim HM (2016) Assessment of the prevalence and risk factors of ophthalmoplegia among diabetic patients in a large national diabetes registry cohort. BMC Ophthalmology 16: 118.

19. Watanabe K, Hagura R, Akanuma Y, Takasu T, Kajinuma H, et al. (1990) Characteristics of cranial nerve palsies in diabetic patients. Diabetes Research and Clinical Practice 10: $19-27$.

20. Agostino R, Cruccu G, Iannetti GD, Innocenti P, Romaniello A, et al. (2000) Trigemina small-fibre dysfunction in patients with diabetes mellitus: a study with laser evoked potentials and corneal reflex. Clinical Neurophysiology 111: 2264-2267. 
Li Q(2020) Function of small-fiber assessed with contact heat evoked potentials in patients with diabetic neuropathy

21. Lajmi H, Hmaied W, Ben Jalel W, Chelly Z, Ben Yakhlef A (2018) Oculomotor palsy in diabetics. Journal Francais d'ophtalmologie 41: 45-49.

22. Said G (2013) Diabetic neuropathy. Handbook of Clinical Neurology 115: 579-589.
23. Tu MC, Chang YY, Lin TK (2010) Recurrent multiple cranial neuropathies in a diabetic patient. Acta Neurologica Taiwanica 19: 208-212.

24. Al-Moallem MA, Zaidan RM, Alkali NH (2008) The sympathetic skin response in diabetic neuropathy and its relationship to autonomic symptoms. Saudi Medical Journal 29: 568-572.

Copyright: $@ 2020 \mathrm{Li}$ Q. This is an open-access article distributed under the terms of the Creative Commons Attribution License, which permits unrestricted use, distribution, and reproduction in any medium, provided the original author and source are credited. 